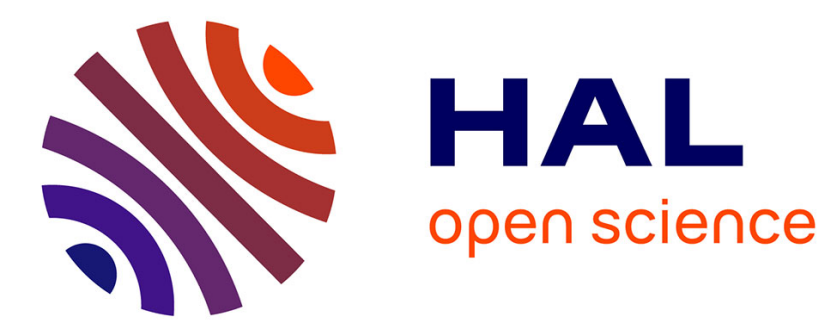

\title{
Remarks on the scaling laws in copolymer mesophases
}

J. Rault

\section{To cite this version:}

J. Rault. Remarks on the scaling laws in copolymer mesophases. Journal de Physique Lettres, 1979, 40 (18), pp.485-491. 10.1051/jphyslet:019790040018048500 . jpa-00231672

\section{HAL Id: jpa-00231672 https://hal.science/jpa-00231672}

Submitted on 1 Jan 1979

HAL is a multi-disciplinary open access archive for the deposit and dissemination of scientific research documents, whether they are published or not. The documents may come from teaching and research institutions in France or abroad, or from public or private research centers.
L'archive ouverte pluridisciplinaire HAL, est destinée au dépôt et à la diffusion de documents scientifiques de niveau recherche, publiés ou non, émanant des établissements d'enseignement et de recherche français ou étrangers, des laboratoires publics ou privés. 


\title{
Remarks on the scaling laws in copolymer mesophases
}

\author{
J. Rault \\ Laboratoire de Physique des Solides, Université Paris-Sud, 91405 Orsay, France
}

(Reçu le 9 mai 1978, révisé le 9 juillet 1979, accepté le 20 juillet 1979)

\begin{abstract}
Résumé. - On montre que certains phénomènes observés dans les mésophases de block-copolymères fondus et gonflés peuvent être expliqués par la présence simultanée de déformations de contraction d'une partie de la chaîne et d'extension de l'autre partie. Le modèle proposé dans cet article diffère des modèles de Meier et de Gennes essentiellement par le fait que l'énergie de surface est considérée comme une petite perturbation.
\end{abstract}

\begin{abstract}
We show that several properties of bulk and swelled block-copolymers can be explained by the simultaneous presence of a stretching of one part of the polymer chain and a contraction of the other part. The model proposed in this paper differs essentially from the theories of Meier and de Gennes by the fact that the surface energy can be considered as a very small perturbation.
\end{abstract}

1. Introduction. - A-B Block-copolvmers of sufficient molecular weight give micropnase separation [1]. The two phase structures due to the intra-chain segregation are :

a) spheres of one component dispersed in a matrix of the other component ;

b) rods of one component parallel to each other dispersed in a matrix of the other component;

c) and alternating lamellar arrangment of the two components.

In these organized domain structures, called mesophases, all the junction points linking the two different parts A and B of the same chain are localised on a surface separating the two domains. The frontier between the two phases is in general very well defined, as are the dimensions of the domains. This can be confirmed by electron microscopy and low angle $\mathrm{X}$-ray diffraction $[2,3]$. The sharpness of the interface depends obviously on the repulsive interaction between unlike A and B segments but also on the preparation of the mesophase (temperature treatment and solvent casting...).

Meier [3] attempts to predict the domain dimensions of the mesophases made with block-copolymer melts. Noting the analogy between the random flight statistics and the heat diffusion with boundary values he arrives at the conclusion that the polymer chain is stretched on both sides of the interface. Figure 1 shows the deformation of the two parts of the chain (Dumbell model) assumed in the theory of Meier. The

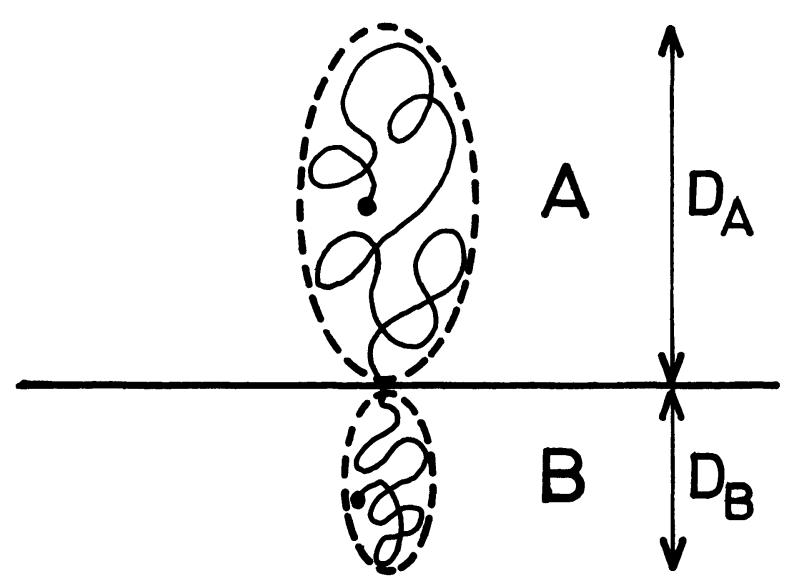

Fig. 1. - The two parts of the copolymer chain are stretched The stretching is due to the fact that placing the junction segment between the two parts A and B on a reflecting surface is a constraint which restricts the number of conformations available to the chain (Meier model).

placement of the linking segment of the two parts $A$ and $B$ is a constraint which restricts the number of configurations available to the chain and therefore a concomitant reduction of chain entropy. The balance between the entropy of the chain which tends to decrease the stretching of the chain and the surface energy which tends to increase the stretching of the chain gives the equilibrium deformation of the polymer coil. In the peculiar case where one part of the chain is much longer than the other, Meier gives the 
dependence of the domain dimension $D_{i}$ of the form :

$$
D_{i}=N_{i}^{\alpha}\left(\frac{\sigma a^{2}}{T}\right)
$$

with $\alpha \sim 0.5$ to 0.65 depending on the value of the surface energy, and $\sigma$ the surface energy.

Recently, de Gennes [4] discussed some scaling laws for a system block-copolymer AB + Solvent $S$, $\mathrm{S}$ being a good solvent of one sequence $\mathrm{B}$ only. One again expects to find lamellar structures, each lamella containing a pure A sheet, covered by two layers $(B+S)$ and then surrounded by an $S$ region. The main assumption of this treatment was that the interfacial energy is high : then both parts of the chain are stretched.

Several objections can be raised concerning these theories.

a) The experimental results of Gervais [7] cannot be interpreted in terms of stretching of both parts $A$ and B of the block-copolymer chain. In some cases, the addition of a preferential solvent increases the thickness of one domain, decreases that of the other domain, and decreases the total long period of the system.

The dimensions of this lamellar (or cylindrical) domain is found for a critical value of the solvent concentration to be less than the unperturbed end to end value of the part of the chain. It is very likely that in this case this part of the chain is contracted.

b) The temperature dependence of the domain dimensions can be estimated only if the temperature dependence of the surface energy and of the unperturbed dimension $\left\langle r_{0}^{2}\right\rangle$ are known. For various polymers the mean square end to end distance $\left\langle r_{0}^{2}\right\rangle$ decreases when the temperature increases [6], the order of magnitude of this variation is :

$$
\frac{\partial \log \left\langle r_{0}^{2}\right\rangle}{\partial T} \sim-1 \times 10^{-3}
$$

$\left(-1.2 \times 10^{-3}\right.$ for polyethylene). This leads to a fictive diminution of the bond length $a$ such that $\left\langle r_{0}^{2}\right\rangle=N a^{2}$. As the dimensions of the domains vary like $a$, we obtain $\Delta D / \Delta T \sim-D \times 10^{-3}$ for $D \simeq 250 \AA$, this value is equal to $-\frac{1}{10} \AA . T^{-1}$ very near the experimental value. Therefore, for this case at least the variation of the domain with the temperature can be explained without invoking any surface energy effect, and the Meier formula need not be correct even when giving the good dependence on the molecular weight.

c) In melt copolymers, in which $N_{\mathrm{a}} \sim N_{\mathrm{b}}$, the domain dimensions are of the order of magnitude of the unperturbed dimension $\left\langle r_{0}\right\rangle$ of the ideal chain. For example [7], in polystyrene-polyoxyethylene copolymers of molecular weight

$$
P_{\mathrm{PS}}=22000 \text { and } M_{\mathrm{POE}}=20400
$$

the dimensions are $D_{\mathrm{PS}}=130 \AA, D_{\mathrm{PEO}}=115 \AA$ and $r_{\mathrm{PS}}=100 \AA, r_{\mathrm{POE}}=110 \AA$. In this case the surface energy is not high enough to conspicuously stretch the both chains. The application of the Meier or the de Gennes formula would give an estimate of the surface energy such that :

$$
D_{\mathrm{a}} \simeq N a^{2 / 3} a \cdot\left(\frac{\sigma a^{2}}{T}\right)^{1 / 3} \sim N a^{1 / 2} \cdot a
$$

and then :

$\frac{\sigma a^{2}}{T} \sim \frac{1}{\sqrt{N a}} \sim \frac{1}{30} \quad \sigma \sim 0.5 \mathrm{dyne} / \mathrm{cm}^{2}$ for $a \sim 2 \AA$.

Another example of chain contraction is the following. In melt PS-PEO copolymer at $80^{\circ} \mathrm{C}$, of molecular weight $M_{\mathrm{PEO}}=10000$ and $M_{\mathrm{PS}}=22000$, the measured radius of the sphere containing the PEO block is $140 \AA$, this is exactly twice the end-to-end dimensions of the ideal PEO chain $r_{\text {PEO }}=75 \AA$. As all the chains cannot occupy the centre of the sphere, the PEO coils inside must be compressed. The exact deformation of these squeezed coils is out of the scope of this paper, but very likely the part of the chain near the surface of the sphere is highly compressed, and the part near the centre is not deformed.

The aim of this paper is to suggest that the morphology of the block-copolymers discussed above is ruled by a balance between the two opposite deformations of the unlike parts of the chain : a stretching of one part, and a contraction of the other part. Thus, we choose an assumption which is the opposite of reference [4]; namely, we assume that the interfacial energy is not high.

We restrict our attention to uncharged polymers, and to polymer chains long enough to be considered as Gaussian chains in the unperturbed state, i.e. chains of length greater than $100 \AA$ for chains of characteristic ratio of about $c \simeq\left\langle r_{0}^{2}\right\rangle / n a^{2} \sim 8$.

2. Self consistent calculation of chain size. - For simplicity we take the same monomer size $a$ for both parts of the polymer chain, and we assume that these unlike chains have the same flexibility : the same persistence length. The mean square end to end distances $\left\langle r_{0}^{2}\right\rangle$ of the two chains are $N_{\mathrm{a}} a^{2}$ and $N_{\mathrm{b}} a^{2}$. The case of melt copolymer is very clear, because as noted by Flory the chains A and B are ideal.

2.1 Melt COPOlymers. Lamellar Phases. - The situation is pictured in figure 2 . The coupling between the two opposite deformations of the A and B coils, is due to the fact that all the lattice sites in the A and B region are occupied by the chains. We define $\Sigma$ the interface area per chain ; $\sqrt{\Sigma}$ is the distance between the centres of gravity of two neighbouring A coils and two B coils. With the assumption of complete saturation of A and B as in reference [4], the thick- 

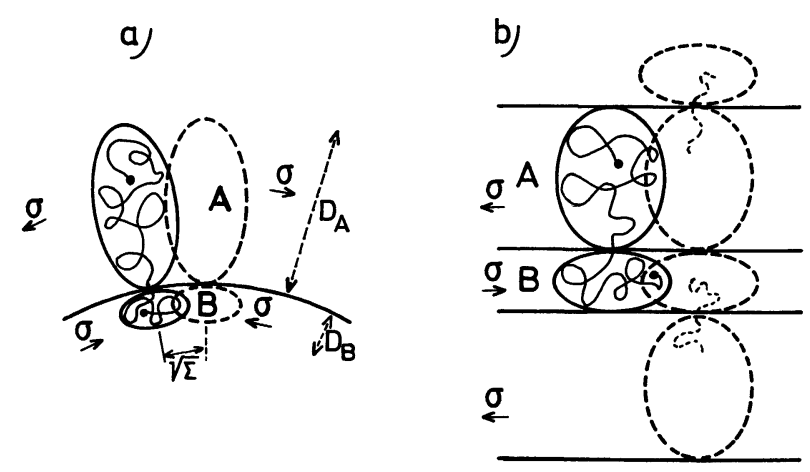

Fig. 2. - Coupling between the stretching energy of the longer part of the copolymer chain, with the contraction energy of the shorter one. For constant density in both domains, the distance between centers of gravity of adjacent A coils and adjacent B coils are equal. The elastic forces $\sigma$ tend to curve the interface $\mathrm{I}$ toward the shortest chains B to give the cylindrical or spherical mesophases in (a) but not in (b).

ness $D_{\mathrm{a}}$ and $D_{\mathrm{b}}$ of domains are related to $\Sigma$, the chain area by the relations :

$$
\begin{aligned}
& \Sigma D_{\mathrm{a}}=N_{\mathrm{a}} a^{3} \\
& \Sigma D_{\mathrm{b}}=N_{\mathrm{b}} a^{3} .
\end{aligned}
$$

a) Large difference in length. - Assuming that the A chain is expanded and the B chain contracted $\left(N_{\mathrm{a}}>N_{\mathrm{b}}\right)$, the free energy of the system is

or

$$
F=\frac{3}{2} k T \frac{D_{\mathrm{a}}^{2}}{R_{\mathrm{a}}^{2}}+\frac{3}{2} k T \frac{R_{\mathrm{b}}^{2}}{D_{\mathrm{b}}^{2}}+\Sigma . \sigma
$$

$$
\frac{2 F}{3 k T}=\frac{N_{\mathrm{a}} a^{4}}{\Sigma^{2}}+\frac{\Sigma^{2}}{N_{\mathrm{b}} a^{4}}+\Sigma \cdot \frac{\sigma}{T}
$$

$R_{\mathrm{a}}$ and $R_{\mathrm{b}}$ are the unperturbed dimensions of the $\mathrm{A}$ and $\mathrm{B}$ chains.

The first term is the stretching energy of the A chain, the second term is the contraction energy of the $B$ chain and the third term the surface energy, with $\sigma=2 \sigma_{0} / 3 k$ which is always positive in melt copolymers, but which can be negative in swelled copolymers.

Minimization of (3) gives :

$$
\Sigma^{4}+\Sigma^{3} \varepsilon_{0}-\Sigma_{0}^{4}=0
$$

with

$$
\begin{aligned}
\varepsilon_{0} & =N_{\mathrm{b}} a^{2}\left(\frac{\sigma a^{2}}{T}\right) \\
\Sigma_{0}^{4} & =N_{\mathrm{a}} N_{\mathrm{b}} a^{8} .
\end{aligned}
$$

The solution of (4) is given in figure 3. There are two regimes, separated by a cross-over for $\varepsilon_{0}=\Sigma_{0}$, that is to say for :

$$
\frac{\sigma a^{2}}{T}=\left(\frac{N_{\mathrm{a}}}{N_{\mathrm{b}}}\right)^{1 / 4} \frac{1}{N_{\mathrm{b}}^{1 / 2}}
$$

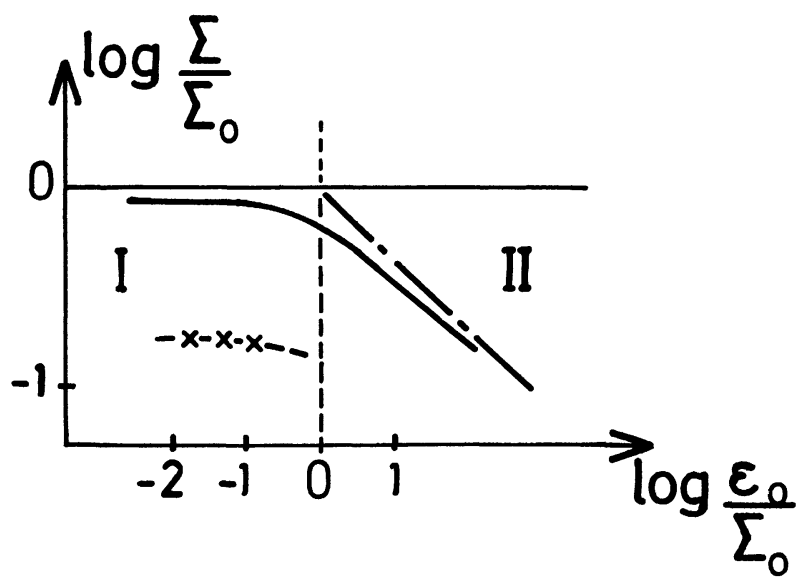

Fig. 3. - Graphical resolution of the equation $\Sigma^{4}+\Sigma^{3} \cdot \varepsilon_{0}-\Sigma_{0}^{3}=0$. The dashed line is the experimental curve of reference [5] for the polystyrene-polyvinylpyridine copolymers, assuming that the surface energy is temperature independent.

The area per chain $\Sigma$ and the domain dimensions are

$$
\Sigma=N_{\mathrm{a}}^{1 / 4} N_{\mathrm{b}}^{1 / 4} a^{2}
$$

$\left.D_{\mathrm{a}}=\left(\frac{N_{\mathrm{a}}}{N_{\mathrm{b}}}\right)^{1 / 4} \cdot N_{\mathrm{a}}^{1 / 2} a\right\}$ in regime $\mathrm{L}_{1}$ for $\varepsilon_{0}<\Sigma_{0}$

$\left.D_{\mathrm{b}}=\left(\frac{N_{\mathrm{b}}}{N_{\mathrm{a}}}\right)^{1 / 4} \cdot N_{\mathrm{b}}^{1 / 2} a\right\}$ (weak surface tension)

and

$$
\Sigma=N_{\mathrm{a}}^{1 / 3} a^{2} \cdot\left(\frac{\sigma a^{2}}{T}\right)^{-1 / 3}
$$

$\left.\begin{array}{l}D_{\mathrm{a}}=N_{\mathrm{a}}^{2 / 3} a\left(\frac{\sigma a^{2}}{T}\right)^{1 / 3} \\ D_{\mathrm{b}}=N_{\mathrm{b}} \cdot N_{\mathrm{a}}^{-1 / 3} \cdot a \cdot\left(\frac{\sigma a^{2}}{T}\right)^{1 / 3}\end{array}\right\} \begin{aligned} & \text { in regime } \mathrm{L}_{2} \text { for } \\ & \varepsilon_{0}>\Sigma_{0}(\text { strong } \\ & \text { surface tension). }\end{aligned}$

Eq. (7a) for $D_{\mathrm{A}}$ coincides with the de Gennes result [4]. Eq. $(7 b)$ is new.

The free energy of the system is :

$$
\begin{array}{ll}
\text { regime } \mathrm{L}_{1} & \frac{2}{3} \frac{F}{k T}=2\left(\frac{N_{\mathrm{a}}}{N_{\mathrm{b}}}\right)^{1 / 2} \\
\text { regime } \mathrm{L}_{2} & \frac{2}{3} \frac{F}{k T}=2 N a^{1 / 3}\left(\frac{\sigma a^{2}}{T}\right)^{2 / 3} .
\end{array}
$$

These two energies are equal at the cross-over.

In regime I, the chain dimensions must verify the assumptions :

$$
\begin{aligned}
N_{\mathrm{a}}^{1 / 2} \cdot a< & D_{\mathrm{a}}<N_{\mathrm{a}} a \\
D_{\mathrm{b}} & <N_{\mathrm{b}}^{1 / 2} a .
\end{aligned}
$$

These inequalities are verified in this regime.

In regime II, eq. (3) assumes the inequality (9) but the physical solution passes continuously from a contracted to a stretched B chain, combining inequa- 
lities $\varepsilon_{0}>\Sigma_{0}$ and inequality $(9 a)$, the eqs. (7) are valid when

$$
\left[\frac{N_{\mathrm{a}}}{N_{\mathrm{b}}}\right]^{1 / 4} \cdot N_{\mathrm{b}}^{-1 / 2}<\frac{\sigma a^{2}}{T}<N_{\mathrm{a}}
$$

contracted B chain exists when :

$$
\left[\frac{N_{\mathrm{a}}}{N_{\mathrm{b}}}\right]^{1 / 4} \cdot N_{\mathrm{b}}^{-1 / 2}<\frac{\sigma a^{2}}{T}<\frac{N_{\mathrm{a}}}{N_{\mathrm{b}}} \cdot \tilde{N}_{\mathrm{b}}^{-1 / 2} .
$$

Therefore the temperature range where contracted $\mathrm{B}$ chain is found in this regime is predicted to be very small. For $N_{\mathrm{a}}=2 N_{\mathrm{b}}$, this temperature range is $\frac{T_{2}-T_{1}}{T_{1}} \sim 0.2$. The temperature variation of the $D_{\mathrm{a}}$ and $D_{\mathrm{b}}$ dimensions of lamellar mesophase $1<\frac{N_{\mathrm{a}}}{N_{\mathrm{b}}}<2$ have not been measured, however several features reported in the following paragraphs tend to support the view that regime II does not exist; involving that the surface energy is smaller than

$$
\left(\frac{N_{\mathrm{a}}}{N_{\mathrm{b}}}\right)^{1 / 4} \cdot N_{\mathrm{b}}^{-1 / 2} \cdot \frac{T}{a^{2}} \text {. }
$$

Estimate of the surface energy. - At the crossover, for $N_{\mathrm{A}}=3 N_{\mathrm{b}}$ and $N_{\mathrm{A}} \sim 10^{3}$, relation ( $5 b$ ) gives $\frac{\sigma a^{2}}{T} \sim 3^{1 / 4} \frac{1}{30}, a \sim 2 \AA, \sigma \sim 0.1 \mathrm{dyne} / \mathrm{cm}^{2}$, this is the same estimate as that obtained by the Meier and de Gennes theory. Therefore, the two regimes are interesting, but we will restrict our attention mainly to regime I.

b) Comparable lengths. - If the length of the A and B parts of the copolymers are nearly equal, the free energy must incorporate the two possibilities (contraction and extension) for each branch. The correct form is :

$$
\frac{2 F}{3 k T}=\frac{N_{\mathrm{A}} a^{4}}{\Sigma^{2}}+\frac{\Sigma^{2}}{N_{\mathrm{A}} a^{4}}+\frac{N_{\mathrm{B}} a^{4}}{\Sigma^{2}}+\frac{\Sigma^{2}}{N_{\mathrm{B}} a^{4}}+\Sigma \cdot \frac{\sigma}{T} .
$$

In this case, for small values of $\sigma$, the chains are not deformed; for high value of $\sigma$, both chains are stretched.

2.2 CylindRical MesophaSe. - The equations expressing complete saturation in cylindrical $\mathrm{B}$ domain and outer A domain are :

$$
\begin{aligned}
\Pi \cdot L \cdot D_{\mathrm{b}}^{2} & =p \cdot N_{\mathrm{b}} a^{3} \\
\Pi . L\left(D_{\mathrm{a}}+D_{\mathrm{b}}\right)^{2} & =p\left(N_{\mathrm{a}}+N_{\mathrm{b}}\right) \cdot a^{3}
\end{aligned}
$$

where $p$ is the number of chains in a section of thickness $L$ perpendicular to the cylinder direction. Then,

$$
D_{\mathrm{a}}=D_{\mathrm{b}}\left(\left(1+\frac{N_{\mathrm{a}}}{N_{\mathrm{b}}}\right)^{1 / 2}-1\right)=D_{\mathrm{b}} \cdot \alpha_{\mathrm{c}}
$$

Here again, two regimes $C_{1}$ and $C_{2}$ are found according to the value of the surface energy compared to the cross-over

$$
\frac{\sigma a^{2}}{T}=\frac{\alpha_{\mathrm{c}}^{1 / 2}}{N_{\mathrm{a}}^{1 / 4} N_{\mathrm{b}}^{1 / 4}} .
$$

Regime $\mathrm{C}_{1} .-$ The surface energy is neglected, the balance between the two deformation energies (stretching and contraction) gives the radius of the cylinders

$$
D_{\mathrm{b}}=N_{\mathrm{a}}^{1 / 4} N_{\mathrm{b}}^{1 / 4} \cdot a \cdot \frac{1}{\alpha_{\mathrm{c}}^{1 / 2}} .
$$

The condition $D_{\mathrm{b}}<N_{\mathrm{b}}^{1 / 2}$. $a$ is written

$$
n<\left[(1+n)^{1 / 2}-1\right]^{2} .
$$

There is no solution to eq. (15a).

For $N_{\mathrm{a}} \gg N_{\mathrm{b}}, D_{\mathrm{b}}$ is of the same order of magnitude as the unperturbed dimension of the coil, and cylindrical mesophase should appear, because the free energy :

$$
\frac{2}{3} \frac{F}{k T}=2\left(\frac{N_{\mathrm{b}}}{N_{\mathrm{a}}}\right)^{1 / 2} \cdot \alpha_{\mathrm{c}}
$$

is lower than the free energy of the corresponding lamellar phase.

The exact verification of the scaling inequalities (9) depend on the precise coefficients in eq. (3). It must be recalled the very crude assumptions mode in our case :

a) The coils A and B have a revolution symmetry around the radius passing by the axis of the cylinders (A oblate coil and B prolate coil). This assumption is not relevant with the symmetry of the hexagonal phase of cylinders.

b) Precise coefficients in eq. (3) should be calculated for comparing the energy of the lamellar and cylindrical mesophase. Casassa [12] calculated the entropy change involved in placing a polymer chain inside a slab, a cylinder and a sphere. As is expected for the same value of the thickness of the lamella and the cylinder, the configuration freedom of the chain is more restricted in the case of the cylinder than in the case of the slab. This fact would lead to a higher energy for the cylindrical phase than for the lamellar phase.

c) The A and B chains do not have in fact the same rigidity $\chi_{\mathrm{a}}=\left\langle r_{0}^{2}\right\rangle / N_{\mathrm{a}} a^{2}$ and $\chi_{\mathrm{B}}=\left\langle r_{0}^{2}\right\rangle / N_{\mathrm{b}} b^{2}$ and the same volume $v_{\mathrm{a}}=a^{3}$ and $v_{\mathrm{b}}=b^{3}$ per monomer unit. Taking into account these two differences we obtain for $D_{\mathrm{b}}$ :

$$
D_{\mathrm{b}}=N_{\mathrm{a}}^{1 / 4} N_{\mathrm{b}}^{1 / 4} \frac{1}{\alpha_{\mathrm{c}}^{1 / 2}}\left(\chi_{\mathrm{a}} \chi_{\mathrm{b}}\right)^{1 / 4}(a \cdot b)^{1 / 2} .
$$


Solutions $D_{\mathrm{b}}<N_{\mathrm{b}}^{1 / 2} b \chi_{\mathrm{b}}^{1 / 2}$ are :

$$
n\left(\frac{\chi_{\mathrm{a}}}{\chi_{\mathrm{b}}}\right)\left(\frac{a}{b}\right)^{2}<\left[\left[1+n\left(\frac{a}{b}\right)^{3}\right]^{1 / 2}-1\right]^{2}
$$

instead of inequality (15a).

High values of $n$ are possible when $\chi_{\mathrm{a}} / \chi_{\mathrm{b}} \leqslant(a / b)$ For the same steric hindrance of the monomer $B$ the greater the rigidity of the $B$ chain, the greater is the possibility of having cylindrical mesophase. Considering the examples of the two copolymers PS-PV2P and PS-PV4P (PS = polystyrene and PVP polyvinyl 2 pyridine and polyvinyl 4 pyridine). This second copolymer gives cylindrical mesophases more easily [9] than the first copolymer. This fact is easily explained by eq. $(15 b)$ because the rigidity of PV4P is 1.4 times higher than that of PV2P. Eq. (15b) shows also that for the same rigidity of the both parts of the copolymer chain cylindrical mesophases are obtained more easily when the longer A chain is thicker than the shorter one, for example by having long side groups.

d) Eq. (11a) implies that in the continuous matrix the A chains are as sketched in figure $4 a$. In fact, as in lamellar mesophases; in this domain the chains coming from different cylinders are interpenetrated as shown in figure $4 b$. Therefore, the second eq. (11) must be replaced by :

$$
\Pi L\left(D_{\mathrm{a}}+D_{\mathrm{b}}\right)^{2}=\left(N_{\mathrm{b}}+\gamma N_{\mathrm{a}}\right) a^{3}
$$

with $1<\gamma<2$.

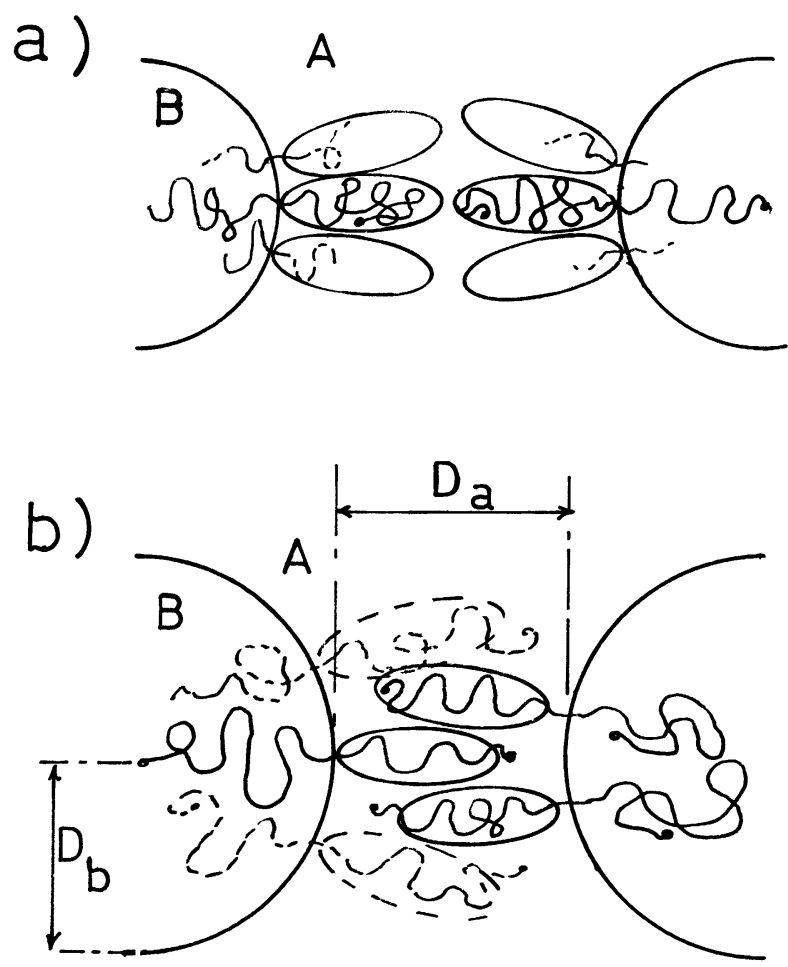

Fig. 4. - Cylindrical mesophase. In the continuous matrix the A chains coming from two adjacent cylinders are not overlapping (a) or overlapping (b). The interpenetration effect in $b(\gamma=2)$ lowers the critical value $n$ for the appearance of regime I; eq. (18).
The two models of the figure 4 correspond to $\gamma=1$ and $\gamma=2$.

The B chain contraction occurs then when :

$$
n<\left[(1+\gamma n)^{1 / 2}-1\right]^{2}
$$

for $\gamma=2$ the cylindrical mesophase occurs for $n \geqslant 4$. Experimentally, this mesophase occurs for $n \gtrsim 1.3$. Therefore, this effect of interpenetration chain cannot explain alone the experimental result, the effects of rigidity and monomer volumes must be taken into account.

Combining the rigidity and interpenetrating effect, it is found that for $a=b, \gamma=2$ and $\chi_{\mathrm{a}} / \chi_{\mathrm{b}}=0.7$ the cylindrical mesophase can appear for $n \geqslant 1.5$.

Regime $\mathrm{C}_{2}$. - For high value of the surface energy, greater than the value at the cross-over, the balance between the surface energy and the A chain stretching energy gives :

$$
D_{\mathrm{b}}=\left(\frac{\sigma a^{2}}{T}\right)^{1 / 3} \frac{N_{\mathrm{a}}^{1 / 3} N_{\mathrm{b}}^{1 / 3}}{\alpha_{\mathrm{c}}^{2 / 3}}
$$

validity of solution $\mathrm{C}_{1}$ and $\mathrm{C}_{2}$ are given in the space $\left(n, \sigma a^{2} / T\right)$ in figure 5. Regime II is valid whatever is the value of $n$; regime I appears only for $n$ higher than the critical value $n_{\mathrm{c}}$.

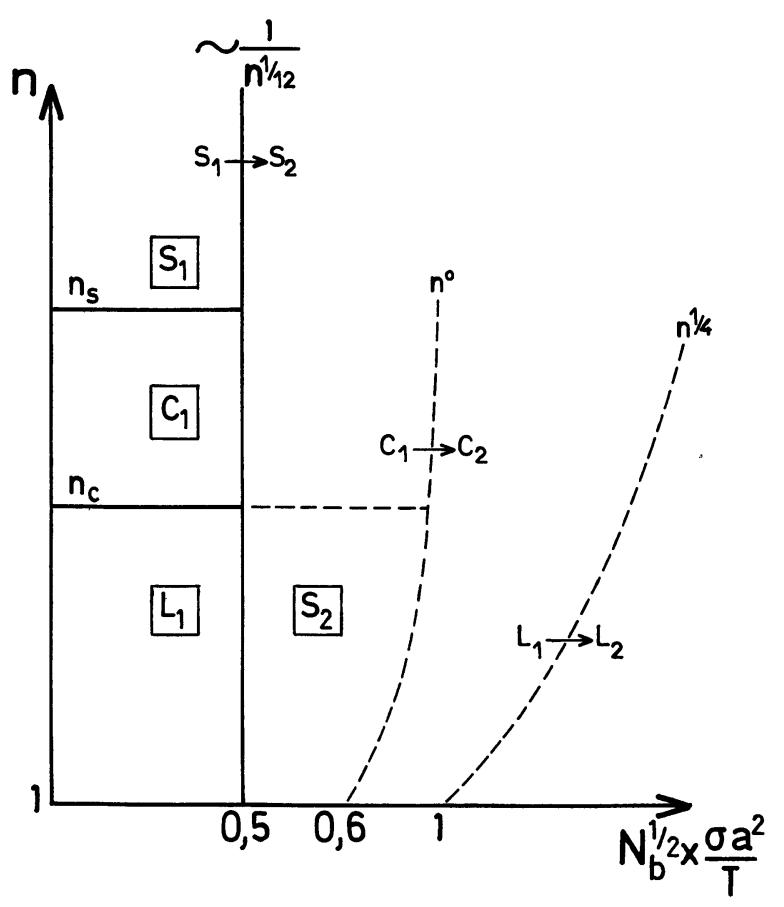

Fig. 5. - Stability of the lamellar (L), cylindrical (C) and spherical (S) mesophases of block-copolymers. The curves $S_{1} \rightarrow S_{2}$, $\mathrm{C}_{1} \rightarrow \mathrm{C}_{2}$ and $\mathrm{L}_{1} \rightarrow \mathrm{L}_{2}$ represent the cross-over between the two regimes I and II. $n_{\mathrm{S}}$ and $n_{\mathrm{C}}$ are the critical values for the appearance of the cylindrical and spherical phases. These values depend on the rigidity of the chains A and B (eq. (15b), $(15 c)$ ) on the interpenetration of the chain (eq. (18)) (and on the relative concentration $C_{\mathrm{b}} / C_{\mathrm{a}}$ of chains in the swelled domains [14]). 
2.3 Spherical MESOPHASE. - Eqs. (11a) and (12a) are replaced by

$$
\begin{gathered}
\left(D_{\mathrm{a}}+D_{\mathrm{b}}\right)^{3} \sim p\left(\gamma N_{\mathrm{a}}+N_{\mathrm{b}}\right) a^{3} \\
D_{\mathrm{b}}^{3} \sim p \cdot N_{\mathrm{b}} \cdot a^{3} \\
D_{\mathrm{a}}=D_{\mathrm{b}}\left(1+\gamma \frac{N_{\mathrm{a}}}{N_{\mathrm{b}}}\right)^{1 / 3}-1=\alpha_{\mathrm{s}} D_{\mathrm{b}} .
\end{gathered}
$$

These equations are not valid in the limit $N_{\mathrm{a}} \gg N_{\mathrm{b}}$.

The two regimes $S_{1}$ and $S_{2}$ are separated by the cross-over

$$
\frac{\sigma a^{2}}{T}=\frac{\alpha_{\mathrm{s}}^{1 / 2}}{N_{\mathrm{a}}^{1 / 4} N_{\mathrm{b}}^{1 / 4}}
$$

which is nearly independent of $n$, and is represented in figure 5 by a vertical line.

The radius of the spherical domains in the two regimes are given by relations $(14 a)$ and $(14 c)$ by doing the transformation $\alpha_{\mathrm{c}} \rightarrow \alpha_{\mathrm{s}}$.

In regime $\mathrm{I}$, the radius $D_{\mathrm{b}}$ does not verify the condition $D_{\mathrm{b}}<N_{\mathrm{b}}^{1 / 2}$. $a$ even for $\gamma=2$. High differences in rigidity and in monomer volume for the $\mathrm{A}$ and $\mathrm{B}$ chains when they exist should be taken into account. The $\mathrm{B}$ chain contraction appears when :

$$
n \frac{\chi_{\mathrm{a}}}{\chi_{\mathrm{b}}}\left(\frac{a}{b}\right)^{2}<\left[\left(1+n\left(\frac{a}{b}\right)^{3}\right)^{1 / 3}-1\right]^{2}
$$

then for a critical value $n_{\mathrm{s}}$ which is always higher than the critical value $n_{\mathrm{c}}$ for example when $\chi_{\mathrm{a}} / \chi_{\mathrm{b}} \sim 0.2$, $a=b, n_{\mathrm{s}}=4$.

2.4 Stability OF THE Different PHASES. - Comparing the energies of the phases $\mathrm{L}, \mathrm{C}, \mathrm{S}$ in the different regions of figure 5 , it turns out that for $\sigma a^{2} / T$ greater than the cross-over value $(13 b)$, the spherical phase in regime $S_{2}$ is more stable than the other phases.

For lower values of the surface energy, the phases $\mathrm{L}, \mathrm{C}, \mathrm{S}$ in regime I are stable in three different domains depending on the value of $n$. Increasing $n$ from 1 , to $n_{\mathrm{c}}$ and then to $n_{\mathrm{s}}$ transitions from $\mathrm{L}_{1} \rightarrow \mathrm{C}_{1}$ and $\mathrm{C}_{1} \rightarrow \mathrm{S}_{1}$ are obtained. That corresponds to the experimental situations. For these reasons we think that in block copolymer mesophase the surface energy is not essential for the stability of the mesophases. No such transitions with $n$ are predicted if the surface energy is greater than the cross-over value. The observation of the transition $L_{1} \rightarrow L_{2}$ by varying the temperature would give an accurate method for measuring the value of the surface energy, but to our knowledge, no transition has been observed by varying the temperature. Finally, it is worth while to note that no transition $\mathrm{L} \rightarrow \mathrm{C}$ is predicted, by varying the temperature.
Conclusion. - The coupling between the two parts of the polymer chain in a copolymer mesophase involves mainly entropic forces in both layers. This situation is quite different from that of amphiphilic systems where electrostatic forces are very important [13], and where entropic forces are less essential in the water layer. In copolymer mesophases, the surface per chain is a function of the molecular weight and does not depend on the temperature, whereas in amphiphilic systems the surface per polar head does not depend on the length of the hydrocarbon chain, and depends strongly on the temperature. In amphiphilic systems transition from.lamellar to cylindrical mesophases are observed when the temperature is changed. This difference in behaviour shows that the scaling laws and stability of the various phases must not be compared.

A balance between stretching energy and surface energy only cannot explain various features observed in copolymer mesophases. But, neglecting the surface energy, the coupling between the stretching and contraction of the two different parts of the copolymer chain explains the following observations :

a) that the domain dimensions are independent of the temperature in concentrated copolymers (in regime I);

b) the sequences of phases lamellar, cylindrical and spherical which appear when the ratios $n=N_{\mathrm{A}} / N_{\mathrm{B}}$. The critical values for appearance of the cylindrical and spherical phases decrease when the rigidity of the longest chain decreases, when that of the shortest chain increases (or when the concentration ratio $C_{\mathrm{b}} / C_{\mathrm{a}}$ in swelled copolymers increases [14]);

c) the absence of phase transition $\mathrm{L} \rightarrow \mathrm{C}$ when the temperature is decreased.

Mean field theory predicts that transitions $\mathrm{L}_{1} \rightarrow \mathrm{S}_{2}$ and $\mathrm{C}_{1} \rightarrow \mathrm{S}_{2}$ appear at the cross-over value

$$
\frac{\sigma a^{2}}{T}=\frac{\alpha_{\mathrm{S}}}{N_{\mathrm{a}}^{1 / 4} N_{\mathrm{b}}^{1 / 4}}
$$

and that the spherical phase $\mathrm{S}$ in regime II is stable for greater values of the ratio $\sigma a^{2} / T$, whatever the ratio $n=N_{\mathrm{a}} / N_{\mathrm{b}}$ is. If not hidden by the glass transition, or crystallization, the observation of these transitions is a convenient mean for measuring the surface energy in copolymer mesophases. Up to now no method has been proposed for getting an order of magnitude of the surface energy. It is obvious that the surface energy depends on the nature of the copolymer chain, and that systems with high surface energy above the cross-over value exist. More experiments on different copolymers systems are needed to know if the properties a-c are general. 


\section{References}

[1] For a general survey of block-copolymers see : Block copolymers, S. Aggawal, ed. (Plenum Press) 1970 Physics of glassy polymers (R. Haward) Appl. Science (1973) p. 548 .

[2] TERISSE, Thèse Strasbourg.

[3] Meier, D., J. Polym. Sci. C 26 (1969) 81.

[4] De Gennes, P. G., Supl. to Solid State Phys. Ehrenreich (Academic Press) 1977.

[5] Grossius, P., Gallot, Y., Skoulios, A., Comptes Rend. Hebd. Séan. Acad. Sci. Paris 270 (1970) 1381.

[6] FloRY, P., Statistical Mechanics of chain molecules (Interscience) 1969.
[7] Gervais, M., Thèse Orléans (1977).

[8] Gervais, M., Gallot, B., Makromol. Chem. 171 (1973) 157.

[9] Gervais, M., Gallot, B., Makromol. Chem. 174 (1973) 193.

[10] Gallot, B., Skoulios, A., Kolloïd-Z. 208 (1966) 375.

[11] Grossius, P., Gallot, Y., Skoulios, A., Makromol. Chem. 127 (1969) 94.

[12] Casassa, E., Polym. Lett. 5 (1967) 773.

[13] Winsor, P. W., Liquid Crystals and Plastic Crystals,' (Chichester) 1974

[14] Rault, J., I.U.P.A.C., Mainz, Septembre (1979). 